\title{
Sea Clutter Target Recognition Based on Modular Neural Network
}

\author{
Hongjie $\mathrm{Yi}^{1,2, a^{*}}$, Guangrong $\mathrm{Ji}^{1, \mathrm{~b}}$, Jinghua $\mathrm{Liu}^{2, \mathrm{c}}$ and Lin Jia ${ }^{1, \mathrm{~d}}$ \\ ${ }^{1}$ Ocean University of China, Qingdao, China \\ ${ }^{2}$ Qingdao Technological University, Qingdao, China \\ ayihongjie@sina.com, bgrji@ouc.edu.cn, 9ljinghua@163.com, djialin@ ouc.edu.cn
}

\section{Keywords: BP neural network; Sea clutter; Modular network; Prediction model}

\begin{abstract}
Sea clutter is a highly nonlinear signal; it has a non-stationary nature both in time and space. Many scholars found that sea clutter data are chaotic. This indicates that the system has some internal rules, but it is difficult to make an analytic formula. The BP neural network with self-organizing fuzzy spatial mapping ability of artificial happens to be a powerful tool for such kind of problem. But it is not enough to get a better result for sea clutter target recognition use an only predict method. So we use modular integrated neural network method in this experiment. The results show that the modular integrated neural network can improve the recognition performance.
\end{abstract}

\section{Introduction of Sea Clutter}

Traditional Sea Clutter Analysis Based on the Mathematical Modeling. Sea clutter usually refers to the ocean surface radar backscatter echoes, is a very complex dynamical phenomena [1]. Sea clutter is a highly nonlinear signal; it has a non-stationary nature both in time and space. We can use mathematical modeling approach to analyze the sea clutter ultimate purpose is to improve the target detection performance. The classical statistical modeling approach is taking the sea clutter as a stationary random process; establishing a distribution model of the process, then performing target detection based on the maximum likelihood guidelines. There are several commonly used sea clutter distribution model, Log-Normal distribution [2], Weibull distribution [3], K distribution [4], $\mathrm{K}+$ Rayleigh distribution [5], etc. But classical detection methods based on statistical models generally require relatively high SCR when it is able to better target detection. In high sea state conditions single statistical distribution models cannot fully described sea clutter characteristics; generally it is difficult to obtain a satisfactory result. Sea ripple can be seen as corresponding to different roughness of surface, so some scholars have proposed the theory with fractal sea clutter nonlinear analysis and modeling [6]. Fractal characteristics of sea clutter has been described [7], multifractal and extended Fractal are often used to describe the sea-clutter [8].

The Chaotic Characteristics of Sea Clutter. Many scholars found that sea clutter data are chaotic when they are analyzing the sea clutter data [9]. Sea clutter is a very complex dynamic system; it is also a highly non-linear time-varying system; it is affected by the sea conditions, weather changes, etc. The chaos characteristics of sea clutter means that there is some kind of internal law. It is showed as correlation between short sequences in time series. Simon Haykin proposed a state space model as follows.

$$
\begin{aligned}
& x(n+1)=f(x(n)) \\
& y(n)=h(x(n))+w(n)
\end{aligned}
$$

In which $x(n)$ is d-dimensional state vector in discrete Time, $f(\bullet)$ is a nonlinear vector function, $y(n)$ is the only detectable value of the system, $h(\bullet)$ is a nonlinear value function, $W(n)$ is additive noise.

This indicates that the system has some internal rules, but it is difficult to make a analytic formula. The BP neural network with self-organizing fuzzy spatial mapping ability of artificial happens to be a powerful tool for such kind of problem. Using a BP neural network to predict a short time chaotic signal trained to build its unknown internal power system. But the chaotic 
properties of the sea clutter will reduce in high sea conditions. At the same time, some scholars questioned the chaotic dynamics of sea clutter [10]. They suggested that sea clutter with positive and negative Lyapunov exponent, but this is not a chaotic criterion. Some scholars point out sea clutter does not have the power rate sensitivity characteristics which is the typical feature of the chaotic signal. Thus, It is not enough to get a better result for sea clutter target recognition use an only predict method. It is very susceptible to interference effects of sea conditions and noise. So we use modular integrated neural network method in this experiment. Two separate modules are the predictive neural network module and the feature classification neural network module.

\section{The Experiment Procedure and Results Analysis}

The Predictive Neural Network Module. We can build a multi-level prediction model as follows.

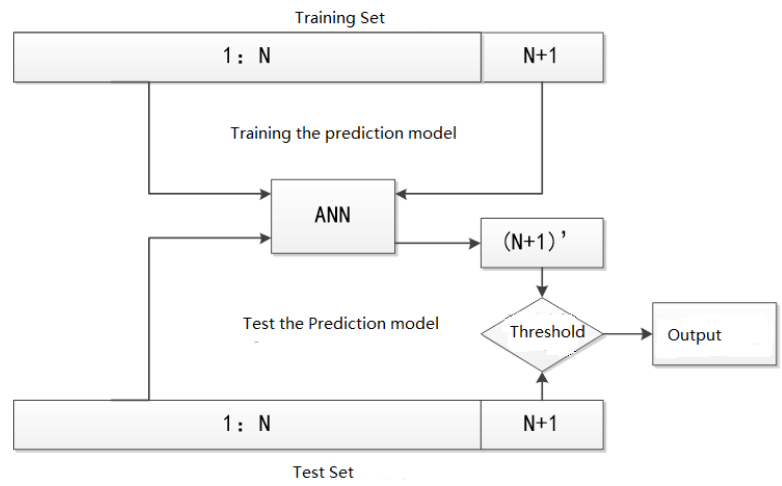

Figure 1. Multi-level prediction model

When there is only sea clutter signal, it should meet this prediction model, the energy of prediction error is small. When the target superposed on a background of sea clutter it should break this prediction model, the energy of prediction error is bigger. By comparison error energy, target detection can be performed under sea clutter.

The sea clutter data used in the experiment is IPIX radar data of Mcmaster University in Canada.We take No. 17 IPIX data for training. The main target appears at the gate 09, the secondary target appears between the gate 08 and gate 11. Frist, construct a series of single-step sequence prediction single hidden layer BP neural network [11]. Then take every 10 data points as a packet to forecast the next data point; use the front 1000 data points for each range gate to make the training set. Finally, use the same network structure and parameters for training and testing. The training condition is the convergence error reaches a given threshold or the training times reached. Now, we compared several network convergence curves and the energy of prediction error. 

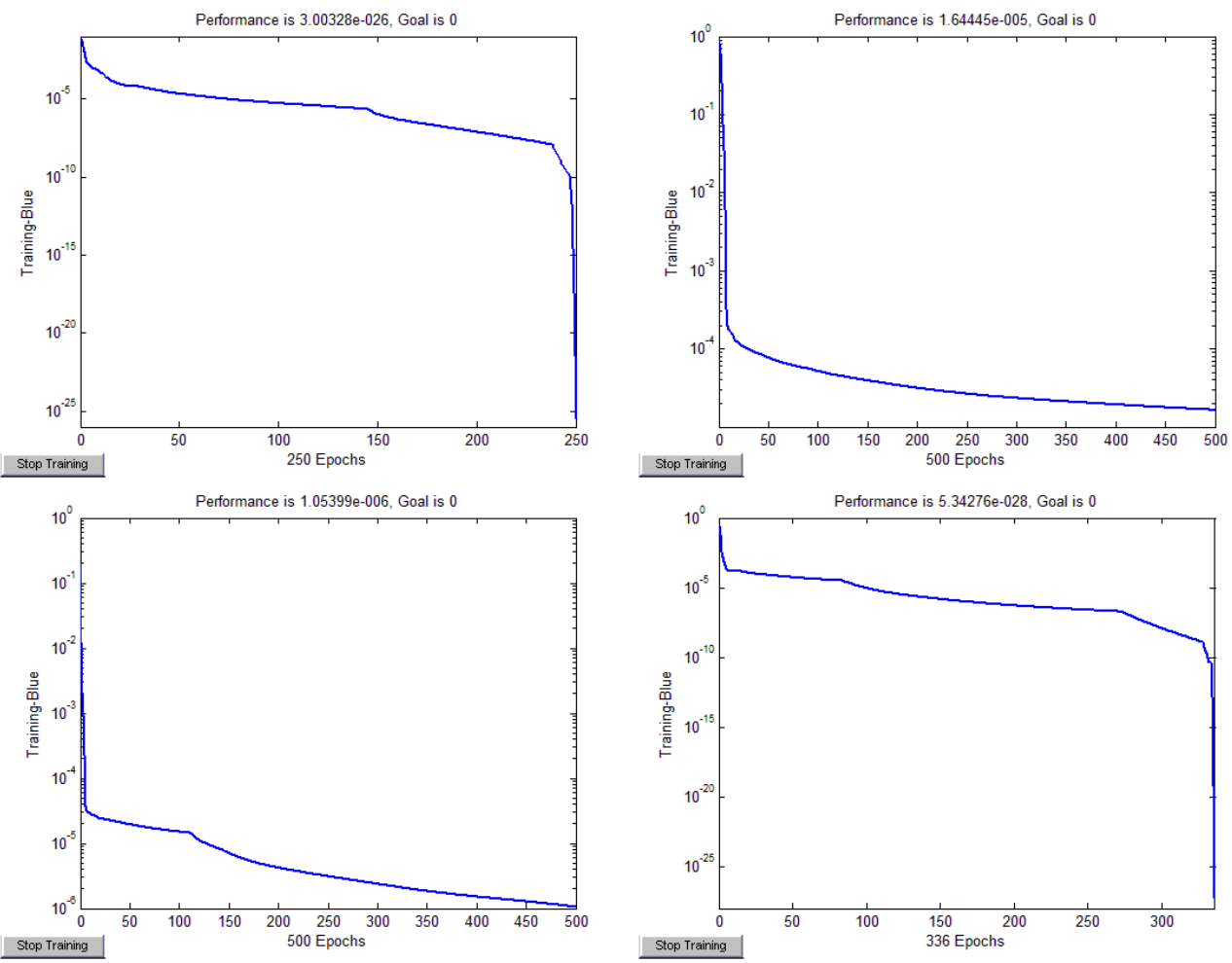

Figure 2. Comparison of network convergence curve (Gate 6, 9, 11, 13)

It can be found, the final convergence error for the gate 6 and gate 13 is very small, and for the gate 9 and 11 is not very small. This shows that the BP neural netwoek has a high prediction accuracy for the gate without target. This reflects the chaotic characteristics are more pronounced for the gate without target.We test the trained networks and compare the energy of prediction error.
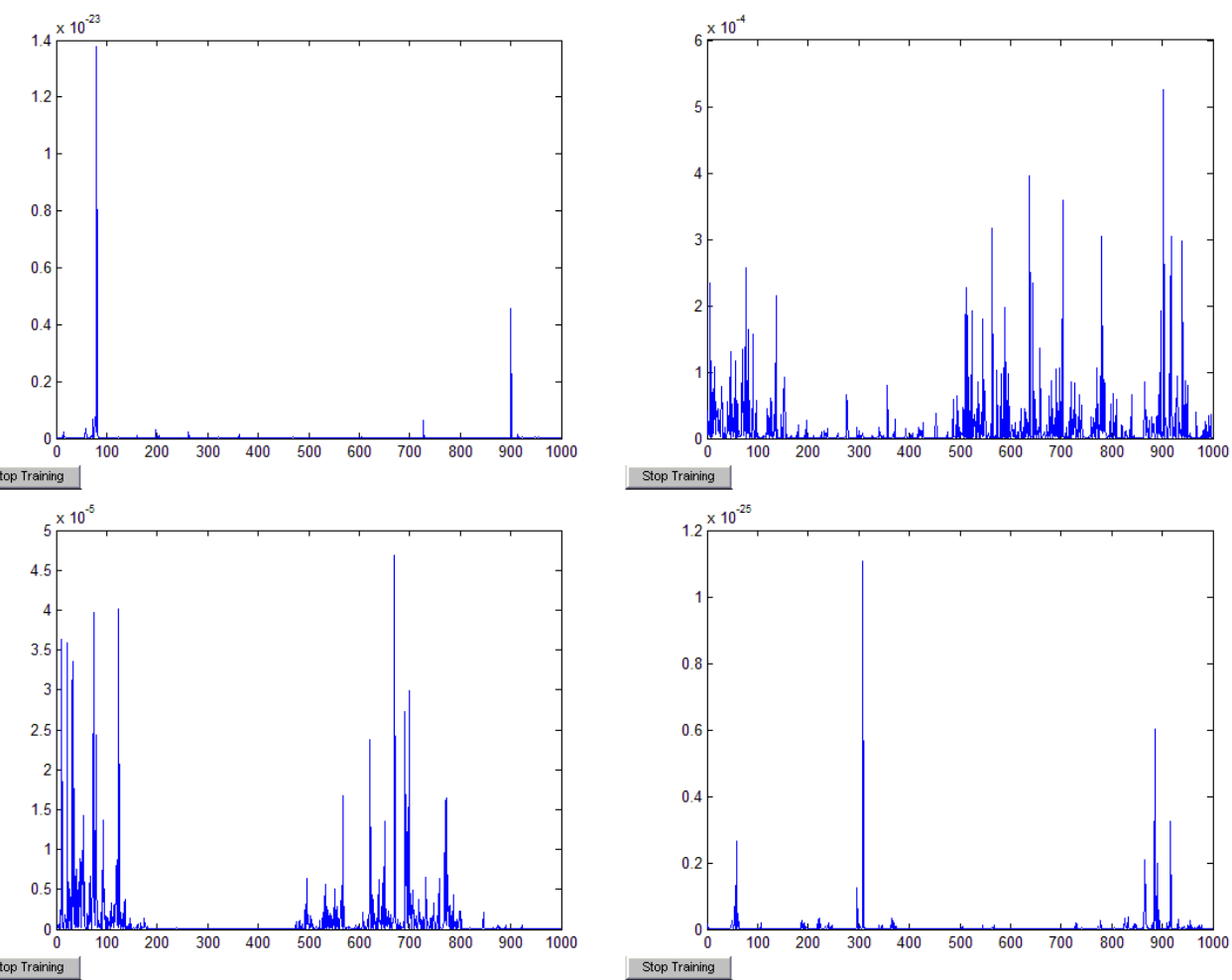

Figure 3. The energy of prediction error of the trained networks (Gate 6, 9, 11, 13) 
Table 1 The MES of the prediction result

\begin{tabular}{|c|c|}
\hline Gate6 & $3.003282 \mathrm{e}-026$ \\
\hline Gate 9 & $2.644454 \mathrm{e}-006$ \\
\hline Gate 11 & $1.053994 \mathrm{e}-006$ \\
\hline Gate 13 & $5.342764 \mathrm{e}-028$ \\
\hline
\end{tabular}

It can be found, the prediction error for the gate 6 and gate 13 is very small, for the gate 9 and 11 is relatively large. So we can compare the the prediction error to determine the existence of a target. Use \# 17,\#30 sea clutter data set to test trained BP neural network. For target recognition the false detection rate is $6.25 \%$, while the false alarm rate is $10.5 \%$.

The Classification Neural Network Module. Although the chaotic characteristics of sea clutter is still controversial, but the recognition for its non-linear characteristic is consistent. Object extraction can be classified by the nonlinear characteristics of sea clutter using neural networks. First, the eigenvectors of the sea clutter are computed. Then a BP neural network is used to classify these feature vectors. It should be noted that, the preprocessing is needed to improve the SNR of the sea clutter data; eigenvector selection should be independent and having classified properties. The process of the feature classification neural network is shown in Fig. 4.

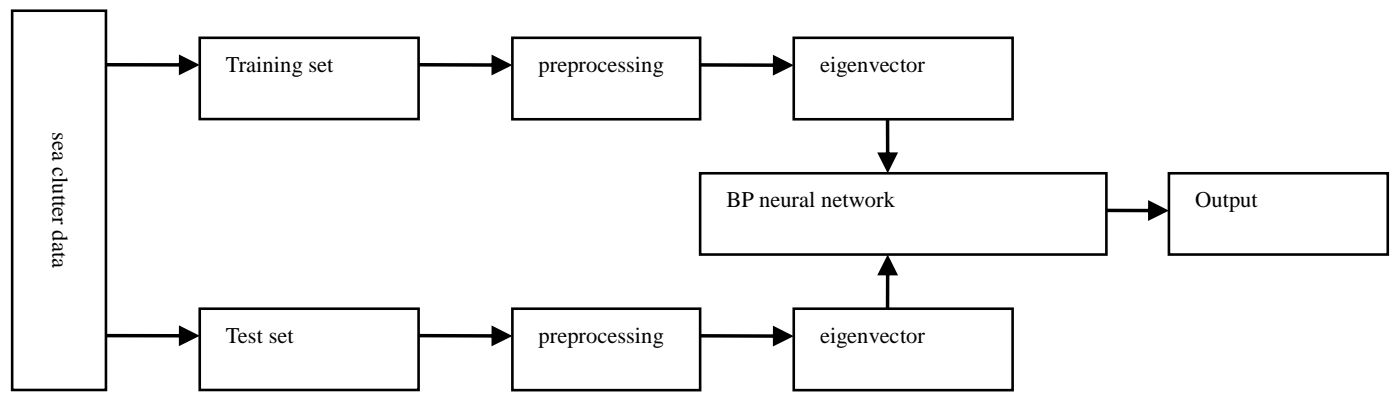

Figure 4. The process of the feature classification neural network

We still take No. 17 IPIX data for analysising. We calculate the eigenvalues for each distance gate, such as amplitude mathematical expectation, variance, FFT transform, power spectral density, autocorrelation, partial correlation, etc. The sesult of Gate 4 is shown in Fig. 5.
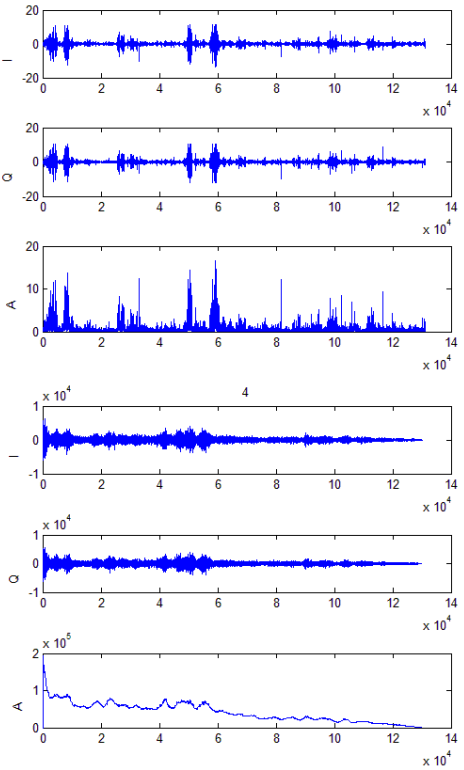
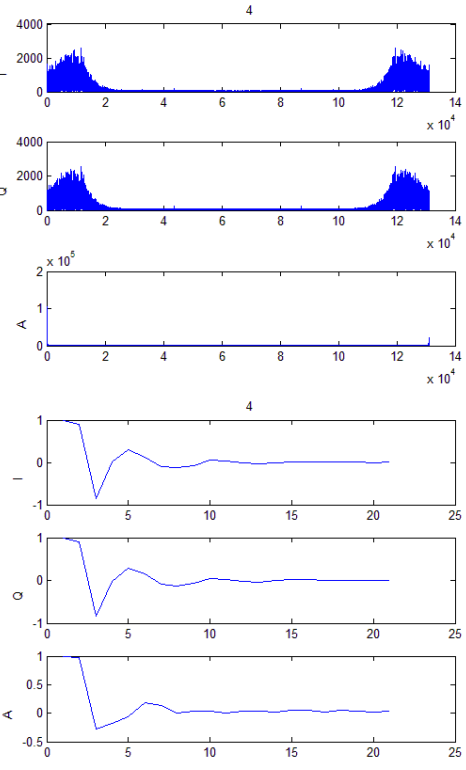


Figure 5. The eigenvalues of Gate 4(amplitude, FFT transform, power spectral density, autocorrelation, partial correlation, mathematical expectation of every gate) 
We use these eigenvalues and prior knowledge such as sea conditions, wind speed, wind direction etc, to structure training set. Use \# 17,\#30 sea clutter data set to test trained BP neural network. For target recognition the false detection rate is $7.5 \%$, while the false alarm rate is $8.25 \%$.

Integrated the Modular Neural Network. These two sea clutter target recognition method based on artificial neural network, both can achieve effective detection of small targets under sea clutter in certain precision. However, due to the complexity of the sea clutter both of them performed not very well separately. We can integrate the two sub-neural networks for target detection. Integrated network structure shown in Fig. 6.

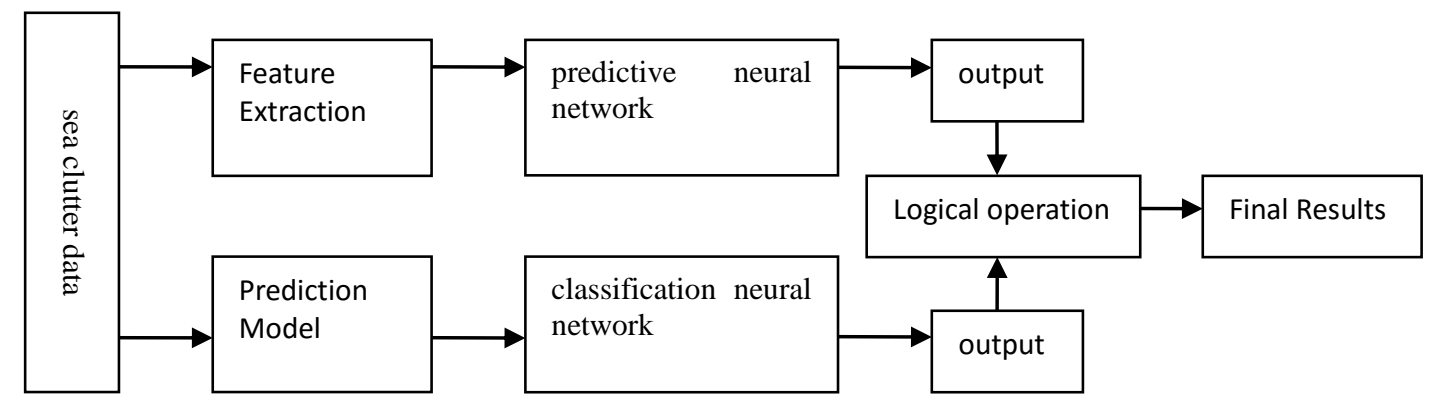

Figure 6. Integrated network structure

The final result can be controlled by adjusting of logic operation. For example, if in practice leakage alarm is more serious.The logic operation should select 'or' operator instead of 'and' operator. This can reduce false detection rate, but in the while the false alarm rate will rise. Experimental results show that the modular integrated neural network can improve recognition performance. It can reduce false detection rate to below $5 \%$.

\section{References}

[1] Unsworth C P,Cowper M R,Mulgrew B et al.Improved surrogate data tests for sea clutter.IEE Proceedings-Radar, Sonar and Navigation.2001,148(3):112-118.

[2] Trunk G V,Georg S F.Detection of targets in non-Gaussian sea clutter.IEEE Trans on Aerospace and Electronic Systems.1970,AES-6(5):620-628.

[3] Fay F A,Clarke J, Peters R S.Weibull distribution applied to sea-clutter.Proc.IEE Conf.Radar,London, 1977:101-104.

[4] Jakeman E,Pusey P N.A model for non-Rayleigh sea echo.IEEE Transactions on Antennas Propag.1976,24(6):806-814.

[5] L Rosenberg, S Watts, S Bocquet, Application of the K+Rayleigh distribution to high grazing angle sea-clutter, International Radar Conference, 2014:1 - 6

[6] Lo T,Leung H,Litva $\mathbf{J}$ et al.Fractal characterisation of sea-scattered signals and detection of ea-surface targets.Radar and Signal Processing, IEE Proceedings F.1993, 140(4):243-250.

[7] Berizzi F,Martorella M,Bertini $G$ et al.Sea SAR image analysis by fractal data fusion.Geoscience and Remote Sensing Symposium, Anchorage,Alaska,2004:93-96.

[8] Y Fan, F Luo, M Li, C Hu, Fractal properties of autoregressive spectrum and its application on weak target detection in sea clutter background, Iet Radar Sonar Navigation, 2015, 9(8):1070-1077

[9] B Mulgrew, CP Unsworth, MR Cowper, S Mclaughlin, SEA clutter \& CHAOS: Improved surrogate-data tests, European Signal Processing Conference, 2015:1 - 4 
[10] C.P.Unsworth, M.R.Cowper,S.McLaughlin,B.Mulgrew. False detection of chaotic behaviour in the stochastic compound k-distribution model of radar sea clutter .2000:296-300.

[11]Hongjie Yi, Guangrong Ji, Haiyong Zheng, Optimal Parameters of Bp Network for Character Recognition, Industrial Control and Electronics Engineering (ICICEE), 2012 $1757-1760$ 